\title{
When Financial Economics influences Physics: The Role of Econophysics
}

\author{
Franck Jovanovic (TELUQ University, Canada, CIRST, and LEO) \\ Rosario N. Mantegna (Palermo University, Italy) \\ Christophe Schinckus (Taylor's University, Malaysia)
}

\begin{abstract}
This paper aims at analyzing the unexpected influence of Financial economics on Physics. The rise of Econophysics, a fundamentally new approach in finance, suggests that the influence between the two disciplines becomes less unilateral than in the past. Methodological debates emerging in Econophysics led physicists to acknowledge that dealing with financial complex systems contributed to a wider modelling of their field. The approach of econophysicists suggests that physicists might try to conceptualize physical phenomena by integrating elements they faced with in Financial economics, and more generally in Economics. Surprisingly, many of econophysicists' argumentations have some methodological similarities with practices used in Financial economics. This paper analyzes the influence of Financial economics on Physics by discussing three examples: (i) out of equilibrium processes, (ii) signal detection and information filtering, and (iii) the role of information in complex systems. It investigates and illustrates what are the methodological changes generated by Econophysics that explain this new influence of finance on Physics. This paper sheds new light on the way finance and economics can improve physics modelling. With this purpose, this article is going one step further in the dialogue between econophysics and economics. Indeed, by investigating the reciprocal influence between the two fields, this methodological paper identifies some areas for a better cross-fertilisation between the fields.
\end{abstract}

Keywords: Econophysics; Minority game; random matrix; financial economics

\section{Introduction}

The influence of Physics on Financial economics, and more generally on Economics is an indisputable fact. A number of writers have extensively highlighted contributions of Physics to the development of Economics and mathematical economics (Boumans, 2004; Ingrao \& Israel, 1990; Le Gall, 2002; Maas, 2005; Ménard, 1981; Mirowski, 1989; Morgan \& Morrison, 1999; Poitras \& Heaney, 2015; Schabas, 1990). Financial economics, and more generally finance, is also subject to the influence of Physics 
(Jovanovic \& Le Gall, 2001; Jovanovic \& Schinckus, 2013a, 2017; Poitras, 2000, 2006; Sornette, 2014). We can mention, among a large variety of examples, the concept of (mechanical) equilibrium, the random walk theory and its variant of the geometric Brownian motion, the Lévy stable distributions, etc. However, the rise of a fundamentally new approach in the 1990s called Econophysics (Jovanovic \& Schinckus, 2017; Mantegna \& Stanley, 1999) suggests that the influence between the two disciplines becomes less unilateral than in the past, and surprisingly Financial economics has an unexpected influence on Physics.

The term econophysics generally refers to the extension of methods and tools traditionally introduced and developed in the field of statistical and theoretical physics to the study of problems commonly considered to fall within the sphere of Economics, and particularly problems in finance ${ }^{1}$. The end of the past century and the beginning of the present one have seen the development of several research areas covering a field between the boundaries of traditional disciplines. Examples are bioinformatics, system biology, cognitive science, network science to cite only a few of them. In this type of disciplines, scholars with a background from two or more well established disciplines start to interact and generate the social and cultural environment needed to develop a new scientific research area characterized by a set of scientific problems, methods, tools and scientific practice. The specificity of each of the two disciplines of Physics and Economics setting the boundary of Econophysics implies an influence from one discipline (or sub-discipline) to the other and vice versa. Precisely, the econophysicists' way of dealing with large quantity of data describing economic, social and financial systems offers a telling example of empirical analyses performed in the absence of micro-founded theories. Today this type of analysis is more common than twenty years ago among scholars of Economics and finance as it is testified by the number of papers dealing with large set of data accepted in academic leading journals of Economics and finance.

Over the past two decades, Econophysics has carved out a place in the scientific analysis of financial markets, providing new theoretical models, methods, and results (Bouchaud, Mezard, \& Potters, 2002; Gabaix, 2009; Jovanovic \& Schinckus, 2017; Lux, 2009; McCauley, 2009; McCauley, Gunaratne, \& Bassler, 2007; Potters \& Bouchaud, 2003; Schinckus, 2018b; Sornette, 2014). The framework that econophysicists have developed describes the evolution of financial markets in a way very different from that used by the current standard financial models. Today, although less visible than Financial economics, Econophysics influences financial markets and practices (Jovanovic \& Schinckus, 2017, p. chap. 5). Many "quants" trained in Statistical physics have carried their tools and methodology into the financial world. According to several trading-room managers and directors, econophysicists' phenomenological approach has modified the practices and methods of analyzing financial data. Hitherto, these practical changes have concerned certain domains of finance: hedging, portfolio management, information filtering of multivariate data, financial crash descriptions, and software dedicated to finance (Bouchaud \& Challet,

\footnotetext{
${ }^{1}$ The theoretical framework of modern finance, which is also called financial economics, was created since the 1950s by American economists (Bernstein, 1992; Fourcade \& Khurana, 2013; Jovanovic, 2008; Poitras, 2006; Poitras \& Jovanovic, 2007; Whitley, 1986).
} 
2014; Casey, 2013; Jovanovic \& Schinckus, 2017, p. chap. 5; Mantegna \& Stanley, 1999; Sornette, 2013; Sornette \& Cauwels, 2015).

From a financial economist's viewpoint, Econophysics aims to provide models that reproduce the statistical behaviors of stock price or return variations, including their extreme values, and then to apply these models to the study of financial products and strategies, such as options pricing, portfolio optimization, market microstructure, design of optimal trading strategies, trading decisions of individual investors, or stock market crashes. The first use in print of the neologism econophysics came in a 1996 article by Stanley et al. (1996). Stanley was also the first to use the word econophysics in a public occasion ${ }^{2}$. The first research community of econophysicists formed in a series of Workshops entirely dedicated to the analyses and modelling of economic and financial systems with methods and tools of statistical and theoretical physics. These were the workshops organized in Budapest (21-27 July 1997), Rome (12-13 March 1998), and Palermo (28-30 September 1998). However, following Kutner and Grech (2008), we can trace the informal birth of the movement to a paper published by Mantegna (1991) that studied the evolution of financial index of the Milan Stock Exchange in terms of Lévy walks. This birth finds its origins in some changes that have occurred in the 1970s and the 1980s in Statistical physics, particularly new developments in the renormalization group theory (Jovanovic \& Schinckus, 2017, p. chap. 3; Lesne, 1998; Lesne \& Laguës, 2012; Stanley, 1999) ${ }^{3}$, and on financial markets, particularly new financial data and the digitalization of financial markets' operations (Jovanovic \& Schinckus, 2017, p. chap. 3).

Econophysics is an example of hybrid discipline. The hybrid nature of econophysics opens room for debates. While some authors (McCauley, 2006; Schinckus, 2010a, 2010b; Stanley, Gabaix, Gopikrishnan, \& Plerou, 2006) emphasize the methodological dissimilarities between the two fields, others (Ausloos, Jovanovic, \& Schinckus, 2016; Jovanovic \& Schinckus, 2013b; Walstad, 2010) explain that there exists a plethora of common conceptual features between these two areas of knowledge. Despite the existence of several conceptual and historical similarities ${ }^{4}$ and some institutional bridges between econophysics and financial economics (e.g., conferences and special issues), the dialogue between the two fields is still difficult. The International Review of Financial Analysis plays a significant role in this dialogue since it is one of the rare journals listed in finance that regularly publishes papers related to econophysics, and the journal already published two special issues devoted to this topic (in 2011 and 2016) ${ }^{5}$.

The influence of physics on the social sciences is not a new phenomenon, and there is a well-documented literature on the history of economics. Numerous papers and

\footnotetext{
2 Actually, Stanley was the first scholar using the term "econophysics" during a conference on the "Physics of Complex Systems" organized in Kolkata (India) in 1995 (Chakrabarti \& Chakraborti, 2010). 3 "The development of [the renormalization group] technique undoubtedly represents the single most significant advance in the theory of critical phenomena and one of the most significant in theoretical physics generally" since the 1970s (Alastair \& Wallace, 1989, p. 237).

${ }^{4}$ We refer here to the works developed by Mandelbrot in the 1960s. See Mandelbrot and Hudson (2004) or Jovanovic and Schinckus (2013b) for further details on this point.

${ }^{5}$ Maintaining an ongoing dialogue between econophysicists and financial economists is an explicit editorial goal of the IRFA since the journal has also a "topic editor" in charge of all papers related to econophysics.
} 
special issues (International Review of Financial Analysis, Journal of Economics Dynamics and Control, European Journal of Physics, Physica A, Quantitative Finance, International Journal of Theoretical and Applied Finance) discuss the contributions of econophysics to finance. Nowadays, Econophysics provides results to most of the areas of capital markets (market microstructure, CAPM, option pricing, statistical properties of price variations, information filtering, financial crashes, portfolio selection, etc.). However, none of them worked on the potential reciprocal influence. This article aims at investigating this unique aspect. How finance and economics can influence physics modelling? With this purpose, this article is going one step further in the dialogue between econophysics and economics. Indeed, by investigating the reciprocal influence between the two fields, this methodological paper identifies some areas for a better cross-fertilisation between the fields.

Econophysics is a fundamentally new approach, although some roots can be traced back to Mandelbrot's work, which had Fama (Fama, 1963a, 1963b) inspired and other financial economists like Fama and Roll (1968, 1971), Blattberg and Sargent (1971), Teichmoeller (1971), Clark (1973) and Brenner (1974)6. Econophysicists are not economists or finance experts taking their inspiration from the work of physicists to develop their discipline, as it has been seen repeatedly in the history of Economics (Mirowski, 1989, 2012). This time, it is physicists who are going beyond the boundaries of their discipline, using their own methods and models to study various problems thrown up by Economics. Such a specific movement has some interesting singularities.

As explained in Jovanovic and Schinckus (2017, p. chap. 4), the singular institutional position of Econophysics -outside Financial economics and in the shadow of Physics - has structured exchanges between econophysicists and financial economists. While it is not hard to understand that this disciplinary structure makes dialogue difficult between Financial economics and Econophysics, it has provided a surprisingly fruitful context for scientific innovations. Precisely, from the observations of economic phenomena, and particularly finance phenomena, Econophysics has given the opportunities to develop new hypothesis, models and methods outside Physics, and then bring them back to Physics. Some examples of such developments are the way econophysicists contribute to the modelling of out of equilibrium processes, signal detection in multivariate systems and information process and aggregation in multi-agents physical systems, as the next section explains.

Such examples suggest that the links between Physics and finance has recently changed. It is worth mentioning that econophysicists' perspective has also changed, defending nowadays a "mutual fertilization"7 between Physics and Financial economics rather than an unidirectional influence of physics on finance, as it was

\footnotetext{
${ }^{6}$ Depict these roots, Econophysics must be separated from Mandelbrot's project (Jovanovic \& Schinckus, 2017). While Mandelbrot and econophysicists arrive at the same result —modeling stock price variations using Lévy stable processes-, Mandelbrot starts his analysis from the stability of stochastic process and the generalized central-limit theorem, explaining why he starts systematically from a stable Lévy distribution, in contrast, econophysicists' starting point is critical phenomena and the results obtained from renormalization group methods, explaining why they start systematically from power-law distributions.

${ }^{7}$ This expression is borrowed from Sornette (2014, p. 1).
} 
common in the past (Sinha, Chakrabarti, \& Mitra, 2016; Sornette, 2014). This article argues that due to its use of financial data newly available, its methods, its methodology and also the new challenges it has faced to by studying financial markets, Econophysics has reversed the traditional relation between Physics and Economics. Section 2 will analyze how Econophysics' research in finance has promoted the investigation of out of equilibrium systems, signal detection and information filtering in multivariate data, and a new role of information in complex adaptive systems. Section 3 will explain the key role of finance in Physics nowadays. Section 4 will conclude about the new influence of Financial economics on Physics.

\section{Three examples of the influence of Finance on Physics}

This section uses three examples in order to show the influence of finance on Physics: the case out of equilibrium processes, the signal detection and information filtering in a multivariate characterization of a complex system, and the elucidation of information role in a complex adaptive system modeled with Statistical physics tools. We discuss hereafter these three cases.

\section{1. The case of out of equilibrium processes}

Physics and Financial economics are both empirical disciplines, but they did not have developed the same way of dealing with data. Such situation generated unexpected contributions on both sides. For discussion or methodological analysis about the influence of econophysics on how financial economists deal with data, see Jovanovic and Schinckus (2017). In this section, we only discuss the influence of Finance and Economics on Physics.

The mainstream approach of Physics is built upon the observation that some quantities are conserved during the time evolution of the studied system. Basic examples are energy, mass, charge, momentum, etc. In other words, major successes of Physics are observed when conservation principles are present in the investigated systems. Another characterizing aspect associated with Physics is that experimental verification of models' predictions is performed in highly controlled experimental settings. Laboratory experiments in Physics are planned in a way to minimize the sources of uncertainty due to uncontrolled events. Experimental set-ups are designed to focus on the studied phenomena. In other words, the phenomena of interest are singled out and the role of any other potential influence is limited as much as possible in the setting. This type of experimental settings implies that the so-called signal to noise ratio is usually high and often not requiring highly sophisticated procedures of statistical validation of the experimental results obtained. It is precisely on this point that finance, and more generally disciplines dealing with open systems whose time evolution is not characterized by conservation laws, suggested new paths in Physics.

In Financial economics, basic concepts most commonly used to build up models are (i) the paradigm of the absence of arbitrage opportunities, which is linked with the efficient market hypothesis, (ii) rational agent, able to subsume all heterogeneities that are present in a real system, that is taking rational decisions by being able to process accurately all information available, and (iii) process of optimization of rational agents 
with respect to a given utility function. The use of these fundamental concepts in economic and financial models is often summarized in the requirement that models need to be correctly "micro-founded". This micro-founded perspective developed by financial economists refers to the necessity to explain phenomena in terms of agents' behaviors. However, the Physics background of econophysicists and their freedom from the need of a micro-founded approach has motivated them to perform empirical analyses also in the absence of a micro-founded theory and/or in the absence of a apriori characterization of the stochastic process of interest. As Schinckus (2014) explained, econophysicists assume that heterogeneous micro-interactions are too complex to be captured through the action of a representative agent so that they do not provide a framework compatible with the classical idea of reduction ${ }^{8}$. When they refer to agents, econophysicists implicitly assume the agents' behavior can be heterogeneous, and that social interactions of heterogeneous agents can be associated with the emergence of global behaviors that are not crucially dependent on the individual choices of economic agents. This phenomenological methodology, reducing the agents' heterogeneity to a collective activity on the macro-scale preserves, is in line with a micro-indeterminism inducing, by coarse-graining, a macrodeterminism. This "coarse-graining situation" is well-known in hard science but not so common in social sciences in which agents are endowed with intentions. The impossibility to define the high number of microscopic configurations for individuals implicitly refers to what we call "the multiple realizability argument" - such approach can be useful for the characterization of "stylized facts" (persistent macro-regularities which cannot be described in terms of microeconomic theory) - among the most studied stylized facts, one can mention: heavy tails of financial distributions, volatility clustering, volume/volatility correlation, absence of autocorrelation in asset return dynamics, emergent phenomena in heterogeneous systems, etc. (Buchanan, 2012; Cont, 2001).

Interestingly, by studying socio-economic systems, some (econo)physicists also start to provide micro-founded framework to their works as witnessed by the increasing number of agent-based modelling in Physics. Methodologically, this modelling takes the form of computerized simulations of a large number of learning and adapting decision-makers and it provides a specific way to study micro-foundations of the statistical regularities that emerge at the macro-level of economic systems (such as stylized facts) ${ }^{9}$. This approach provides a complementary perspective on the macropatterns identified by the usual statistical models used by econophysicists. In doing so, the latter extended their way of modelling the agents' behavior by enlarging the way they characterize the heterogeneity of individual components.

From the empirical side, the accuracy of the estimated quantities and/or of the statistical assumptions done for the modelling of economic and financial systems are typically model dependent and/or depending on the assumptions about the stochastic processes that are assumed to describe the evolution of the system of interest. The investigated system is always an open system which is monitored and described under

\footnotetext{
${ }^{8}$ Nagel explained that "reduction [...] is the explanation of a theory or a set of experimental laws established in one area of inquiry, by a theory usually though not invariably formulated for some other domain" (Nagel, 1961). Reduction is therefore defined through the logical idea according to which a theory can be a definitional extension of another.

${ }^{9}$ For further information about this increasing literature see Schinckus (2016).
} 
specific modelling assumptions that are providing both the interpretative framework of the results obtained and the confidence intervals of the quantities estimated.

The traditional statistical care of Econometrics approaches of economic and financial systems has been progressively imported first in Econophysics studies and through them also in some more traditional area of studies of Physics. For example, the need for a more detailed statistical control of empirical analyses arises for physical systems not presenting manifest conservation laws as (i) interconnected complex systems well described by networked relations or (ii) evolving systems not characterized by an equilibrium that can be interpreted as a thermal equilibrium ${ }^{10}$.

Examples of this type of studies are seen in network science where statistical physicists single out specific links of a network by considering (i) the rejection of a null hypothesis concerning the strength ${ }^{11}$ partitioning of a specific node (Radicchi, Ramasco, \& Fortunato, 2011; Serrano, Boguná, \& Vespignani, 2009) or by performing a statistical test of the over-expression or under-expression of repeated actions that are observed between elements of a bipartite complex system (Tumminello, Micciche, Lillo, Piilo, \& Mantegna, 2011).

In fact, studies performed in Econophysics have shown that the analysis and modelling of physical systems can today start to overcome traditionally self-imposed limitations. Such situation ends up the strong bias of the discipline about the preference for studies of homogeneous systems at a thermal equilibrium and might foster interest towards studies of heterogeneous systems near or out of equilibrium presenting stationary or quasi-stationary statistical regularities. As examples, one can mention studies modelling the growth rate of firms or the evolution of wealth distribution described as a multiplicative stochastic process with fixed boundaries (Levy, Solomon, \& Ram, 1996). The Gibrat's law commonly used by the economic mainstream are clear examples of growth models reaching a stationary distribution that can model both economics and Physics open growing systems. The search for an explanation of the observed power law deviation from the growing lognormal Gibrat's law made clear that the presence of a boundary at low or zero value of the wealth or income is a key aspect for the observation of the power law deviation detected for high values of wealth as mathematically shown in the work of Kesten (1973). The following section will present another illustration of how financial economics can influence modelling practices in physics.

\section{2. Signal detection and information filtering in multivariate characterization of a complex system}

The second example of the influence that Financial economics had on Physics concerns the signal detection and information filtering in multivariate systems, and specifically in the correlation matrix of returns of financial assets. This is a classic

\footnotetext{
${ }^{10}$ It should be noted that the equilibrium discussed here is thermal equilibrium or an equilibrium formally analog to a thermal equilibrium. The classic concept of equilibrium present in economic theory has an origin from Physics but it is a different concept of equilibrium being a form of equilibrium equivalent to the mechanical equilibrium observed in a system of masses and forces.

11 The strength of a node is the sum of the weights of links of the node.
} 
problem in Finance since the introduction of Markowitz portfolio optimization that pointed out the crucial role played by covariance matrix of assets' returns (Markowitz, 1952). One key result of Econophysics concerns the use of random matrix theory (Bouchaud \& Potters, 2011; Conlon, Ruskin, \& Crane, 2009; Laloux, Cizeau, Bouchaud, \& Potters, 1999; Plerou, Gopikrishnan, Rosenow, Nunes Amaral, \& Stanley, 2000) and of filtering methods based on hierarchical clustering (Mantegna, 1999) to detect signals (i.e. underlying regularities), which are present in correlation matrices estimated by using a finite number of records. The use of random matrix theory in the investigation of a correlation matrix has been instrumental to have an unsupervised criterion to discriminate between the information that is distinctively present in the matrix from the one that is indistinguishable from a multivariate random process of given statistics.

While the origins of random matrix theory can be traced back to Hurwitz (1897), Wishart (1928) and then Wigner (1957), its application on correlation matrices is recent and was primarily developed by econophysicists while investigating stock market dynamics. Although, random matrix theory and applications have been and are investigated by disciplines as different as mathematics, physics, and biology (Akemann, Baik, \& Di Francesco, 2011), the approach followed by econophysicists was absolutely original and directly motivated by the aim to achieve a full control of the information carried by the correlation matrix of returns of a portfolio of assets. More precisely, the application of random matrix theory to the modelling of correlation matrices of stock returns has shown that correlation coefficients estimated using a finite number of records (a limitation that is always present in empirical estimations) present two basic distinct types of information (Laloux et al., 1999; Plerou, Gopikrishnan, Rosenow, Nunes Amaral, \& Stanley, 1999). The first type of information can be easily detected in the correlation matrix. Random matrix theory makes clear the nature of this information. It is the information associated with the principal components whose spectral position lies outside the eigenvalues interval where random matrix theory predicts the presence of eigenvalues for random processes characterized as specific stochastic processes (in the most basic setting as independent Gaussian random processes). The second type of information is the information associated with eigenvalues falling inside the eigenvalues' interval predicted by random matrix theory. This type of information may or may not be associated with correlation coefficients estimated in a statistically reliable and unbiased way but, in any case, the information associated has a nature that it is hardly distinguishable from a random pattern (Bun, Bouchaud, \& Potters, 2017). Therefore, extraction of this information can be achieved only with highly sophisticated filtering techniques (Tumminello, Lillo, \& Mantegna, 2010).

The use of random matrix theory and its application on correlation matrices in Econophysics triggered a large amount of activity in Physics dealing with the role of different underlying stochastic processes in the exact determination of the eigenvalues' spectrum. See, for example, the case of Levy processes (Arous \& Guionnet, 2008; Burda, Jurkiewicz, Nowak, Papp, \& Zahed, 2005; Cizeau \& Bouchaud, 1994). Another wide research area of investigation concerns the different methodologies to be used to extract the informative structure of the correlation matrix for both the two sets of information discussed above (Burda, Görlich, Jarosz, \& Jurkiewicz, 2004). The need to extract sound information from the correlation matrix 
or, more generally, from any proximity matrix has motivated statistical physicists to propose several techniques that are successful in the filtering of information from a multivariate set. Examples are the extraction of the minimum spanning tree (Mantegna, 1999) or of the planar maximally filtered graph (Tumminello, Aste, Di Matteo, \& Mantegna, 2005) associated with a correlation matrix or the planar maximally filtered graph associated with a partial correlation matrix. The information extracted can be quantified in a way that is independent of the true correlation matrix by using the Kullback-Leibler entropy, i.e. a well-defined measure of information theory (Biroli, Bouchaud, \& Potters, 2007; Tumminello, Lillo, \& Mantegna, 2007).

The impact of finance in these studies considering the information content of time series (or more generally vectors) can also be seen by considering the progressive import, reconsideration and interpretation of Granger causality (Granger, 1969) within the Physics community. Granger causality is a methodology originally developed in Finance that it is now used in many fields of research. Physics studies have developed nonlinear generalizations of it (Marinazzo, Pellicoro, \& Stramaglia, 2008) and have shown that in the case of Gaussian variables it is equivalent to the more familiar concept of transfer entropy, a concept developed in the field of information theory (Barnett, Barrett, \& Seth, 2009).

This type of knowledge was originally used and discussed in the research performed in Econophysics but it is now knowledge of Statistical physics that can be used in any field of Physics where the multivariate nature of the system evolution is a key aspect of the system. The next section will present the last example we want to discuss.

\section{3. The role of information in a complex adaptive system investigated in Physics}

Our third and last example of the influence that Finance had in the setting of a Statistical physics problem and in fostering new concepts in this research area concerns a dynamical system with many agents that is presenting different phases in its dynamical evolution depending on a control parameter. The investigation of this type of system is the wide investigation of the so-called "minority game" and has a clear origin in a model originally proposed in economics.

Minority game (Challet \& Zhang, 1997) is a stylized version of the "El Farol bar" problem, a well-known problem in game theory, originally introduced by Arthur (1994). In the "El Farol bar" problem, a number of agents wish to take some action, but they will not benefit of the action if the majority of agents do the same. The motivation in economics was to introduce an illustrative example of the process of rational decision between two alternatives, says 0 and 1, of a group of rational agents in the presence of negative externalities. In this setting, there is no self-fulfilling equilibrium and therefore by assuming fully rational use of the public information the system oscillates between states that are always frustrating for the agents. By introducing his model, Arthur was able to show that a suboptimal (economic) equilibrium occurring at each time step around an a-priori optimal allocation of the resource is reached by the system by hypothesizing a bounded rational inductive reasoning of the agents. 
By formalizing the "El Farol bar" model as a minority game, two econophysicists, Challet and Zhang (1997), inspired by financial analogies, defined a stylized model to be investigated and analyzed with tools and concepts of Statistical physics, particularly phase transition, control parameter, and order parameter. Minority game is a telling example how Econophysics provides a solution to a problem that was created in economics and was not tractable in terms of classic game theory. As Arthur explained, "economists didn't quite know what to make of [my paper presented at the January 1994 American Economic Association meeting]. My colleague at Santa Fe, Per Bak, did know however. He saw the manuscript and began to fax it to his physics friends. The physics community took it up, and in the hands of Challet, Marsili and Zhang, it inspired something different than I expected - the Minority Game. El Farol emphasized (for me) the difficulties of formulating economic behavior in ill-defined problems. The Minority Game emphasizes something different: the efficiency of the solution" (B. W. Arthur, 2004). The investigation and the results obtained were of great interest with many key concepts of Statistical physics observed in the stylized model and with key novelty about the order parameter. A phase transition is observed in the model between two distinct regimes of the deterministic time evolution of the system. More noteworthy, a quantity that may act as an order parameter of the phase transition (i.e. the quantity that is discriminating between the two distinct phases observed in the system) is directly expressed in terms of the information that can be extracted from the publicly available time series of the aggregated state of the system (that is the number of agents that decided state 0 or 1 at each time step, for instance to buy or to sell) (Challet, Marsili, \& Zhang, 2013; De Martino \& Marsili, 2006).

In other words, this is a stylized model of bounded rational heterogeneous agents that can be solved with state-of-the-art tools and methods of Statistical physics. Specifically, the model has been investigated and solved by adapting and using tools of statistical physics originally developed to investigate and model disordered physical systems, primarily glasses and disordered magnets (Mézard, Parisi, \& Virasoro, 1987). To fully evaluate the originality of the approach stimulated by the setting of the minority game it is worth noting that "the stochastic equations of minority games do not evolve towards an equilibrium state, so a proper mathematical analysis requires solving their dynamics. This task was found to be surprising non-trivial" (Coolen, 2005). Two methods have been primarily used to obtain exact results. They are the so-called replica trick and the generating functionals approach (Chakraborti et al., 2015).

The solution obtained shows the existence of two distinct phases. The system will be in a given phase according to the value of the control parameter that in the simplest version of the game is $\alpha_{c}=2 \mathrm{~m} / \mathrm{N}$ where $\mathrm{m}$ is the number of records each agent uses to select the strategy to be used to make the binary choice and $\mathrm{N}$ is the number of agents participating to the game. The two phases are different with respect to the ergodic or non-ergodic nature of the deterministic evolution. The phase with $\alpha<\alpha_{c}$ is non-ergodic and the global efficiency of the system is controlled by the initial conditions whereas the phase with $\alpha>\alpha_{c}$ is ergodic and information can be extracted by the agents from the publicly available information. The measure of the degree of predictability used in minority game is 


$$
H=\frac{1}{2^{m}} \sum_{\mu=1}^{2^{m}} E\{A(t) \mid \mu\}^{2}
$$

where $A(t)$ is the time series of the outcome of the global state of the systems fluctuating around zero and $\mu$ is the set of sequences used to select the strategy agents are using during the game. In the non-ergodic phase $E\{A(t) \mid \mu\}=0$ for all $\mu$ and hence $\mathrm{H}=0$. In the other phase $\mathrm{H} \neq 0$ and non-trivial predictions can be done about the $A(t)$ outcome. We have already noticed that that $H$ acts like a 'physical' order parameter.

When initial conditions are set randomly the efficiency of the system, i.e. the amount of fluctuations $\sigma^{2}=E\left\{A^{2}\right\}$ from the optimal allocation $A=0$ of choices is minimized when $\alpha \cong \alpha_{c}$ indicating that the control parameter $\alpha$ is useful to select the condition that maximize the overall suboptimal allocation of resources in the system.

The study and the achievement of the exact solution of minority game has demonstrated that theoretical methods introduced to investigate disordered systems in statistical physics can be used to model, and in some cases solve, resource allocation problems occurring in a large population of agents taking myopic decisions in a decentralized setting. General examples of this type of extensions are different version of minority games, the so-called Kolkata paise restaurant problem, the parking space problem, the stable marriage problem and, more generally, recommendation problems trying to guess the preference lists of items to agents based on partial information in a heterogeneous setting (Chakraborti et al., 2015).

Created in order to analyze an economic problem, improved by studying finance issues, minority game has been applied back to Physics and some related fields in order to model several problems. For instance, it is used in radio engineering and computer science in order to improve wireless networks (Mähönen \& Petrova, 2008; Sungwook, 2014), secondary users battery life and network performance (Elmachkour, Daha, Sabir, Kobbane, \& Ben-Othman, 2014; Elmachkour, Sabir, Kobbane, Ben-Othman, \& El Koutbi, 2014), or to improve coordination in wireless sensor networks (Galstyan, Krishnamachari, \& Lerman, 2004). In computer science, minority game is used to improve the reconfigurable multi-core processors (Shafique, Bauer, Ahmed, \& Henkel, 2011) or heterogeneous Delay Tolerant Networks (Sidi, Chahin, El-Azouzi, De Pellegrini, \& Walrand, 2013). It is also used in order to improve energy management system (EMS) of buildings (Zhang, Wu, Huang, \& Yu, 2012). According to Mähönen and Petrova (2008, p. 100), it could also be applied for studying the behaviors of flocks of birds.

It is worth noting that economics and finance are naturally dealing with systems where information is processed by rational agents and/or agents with bounded rationality, who are taking their decisions. By opposition, this is not the case in Physics. In Physics, while various forms of information are also investigated, the investigation is primarily limited to the use of information as a tool to quantify the degree of disorder present in the system. For example, in dynamical systems information production of a symbolic sequence associated with the time evolution of the dynamical system is describing whether the system is evolving in an attractor characterized by a simple 
structure or rather characterize by a complex structure as in the case of the so-called "strange attractors".

Econophysics studies of the "minority game" have shown that studies of stylized physical system of economic, social or financial origin can investigate the use, spreading and aggregation of information in a way that is rigorous, explanatory and highly informative about the investigated system.

\section{The key role of financial markets and practices and Econophysics}

The previous examples show that, while Econophysics at the beginning was driven by the application of Physics to Financial economics, in the recent years the opposite influence has been observed. This section aims at identifying some distinctive characteristics of finance (as science and sector) that explain the key role of this field in this new influence of Financial economics on Physics. We already mentioned the traditional statistical care of econometrics approaches. Two linked reasons are also important: the increasing number of financial data and the particularity of phenomena studied by econophysicists.

One distinctive characteristic of finance is the numerous available data, which has played a key role in the discovery of new phenomena and in scientific developments, and particularly in Physics. One well-known example is Louis Bachelier (1900) who was trained in mathematical physics. Bachelier's work is generally mentioned in order to show how Physics influences finance, particularly by proposing the first mathematical model for pricing Premium contract (called "Prime"), which was a conditional forward contract close to option. However, it is only the second step of Bachelier's reasoning. The first step shows, on contrary, the influence of financial data on Physics (Ben-El-Mechaiekh \& Dimand, 2018; Jovanovic, 2012; Samuelson, 1973; Taqqu, 2001) that allowed Bachelier to introduce the continuous-time probabilities, the theory of Brownian motion, to develop the mathematical theory of diffusion (trajectories of Brownian motion), and to solve the parabolic diffusion equation five years before Albert Einstein (1905). Financial data was the major starting points of Bachelier and the foundations of all of his demonstrations. Precisely, the numerous available financial data gave to him the opportunity to demonstrate the equivalence between results obtained in discrete time and in continuous time ${ }^{12}$.

What was true for Bachelier is still true today. Financial databases are nowadays the largest bases for social phenomena due to the progressive automation of financial markets. Precisely, finance was the first research area of economics where large amount of digitized data started to be stored, processed and analyzed. Since the end of the 1970s, all the major financial markets have been progressively automated thanks to computers ${ }^{13}$. In addition, some markets, like the foreign exchange market,

\footnotetext{
12 It is worth mentioning that stock market prices on Paris stock exchange were published and largely available because it was the law. Data had to be published by the agents de change (i.e. the official stockbrokers) whose nomination was approved by the Minister of Finance and appointed by decree of the President of the Republic. One of their obligations was to record and publish the stock market prices. In his thesis, Bachelier used the data between 1894 and 1898 (Bachelier, 1900, p. 58).

13 In 1977, the Toronto Stock Exchange became the first stock exchange to be fully automated. Then, the Paris Stock Exchange (now Euronext) imported Toronto's system and became fully automated at
} 
became active 24 hours a day with electronic trading. Automation has allowed all transactions and all prices quoted to be recorded, leading to storage of a huge amount of financial data. Moreover, since the 1990s, use of computers has enabled the development of high-frequency transactions, and therefore the creation of highfrequency data (also called "intraday" data) ${ }^{14}$. Previously, statistical data on financial markets were generally made up of a few values per day obtained by the average price, minimum, maximum and often the last quotation of the day. Nowadays, with the recording of all orders and transactions, all prices quoted and tens of thousands of transactions are conserved every single day (Engle \& Russell, 2004) for highly liquid assets.

Due to the computerization of finance and the automation of financial markets, Financial economics becomes a discipline that produces a high rate of scientific data and information. Although finance is not the only one discipline in this case, the explosion of financial data comes closer to the standards to which statistical physicists generally work. Precisely, in an economic system, one initial work was limited to analyze time series of order of magnitude $10^{3}$ records, and nowadays with highfrequency data and algorithmic trading, one may typically consider order $10^{8}$ records. By comparison, macroscopic samples in physical systems contain a huge number of interacting subunits, as many as Avogadro's number, $6 \times 10^{23}$ (Stanley \& Plerou, 2001, pp. 563-564). Consequently, Physics is no more the discipline that is producing the more empirical data, and econophysicists interested in finance have new opportunities for discovering new phenomena and regularities. The increasing quantities of data, the availability of intraday data, and the computerization of financial markets led to notable changes in techniques for detecting new phenomena (cf. Power law, etc.). Intraday data brought to light new phenomena that could not be detected or did not exist with monthly or daily data. Among these are strategic behaviors that influence price variations (Jovanovic \& Schinckus, 2017, p. 61).

Beyond the increasing importance of computers, the influence of Financial economics on Physics can actually be explained through a methodological perspective on the way physicists applied their knowledge to Economics, and consequently the key role of Econophysics in this process. The role of analogies explains that from a physicist's point of view, Econophysics can be perceived as an analogical and idealized extension of Physics models, tools and concepts that appear to be theoretically, empirically and logically justified. This explains, from a methodological perspective, how econophysicists can justify the transfers from Physics to Financial economics. Interestingly, this analogical extension of Physics would not be possible without the specificities of the field in which Econophysics has mainly been developed: Financial economics. Analogies are not necessary unilaterally implemented when, for instance, a formalization of the field 1 is used to describe the field 2 (F1 $\rightarrow F 2$ ) simply because the perfect synonymy between two systems is impossible. Consequently, such

the end of the 1980s. These changes occurred for NASDAQ between 1994 and 2004, and later for the NYSE in 2006 with the introduction of the NYSE hybrid market. The Tokyo Stock Exchange switched to electronic trading for all transactions in 1999.

${ }^{14}$ High frequency trading, which is based on high-frequency data was virtually unknown ten years ago, yet it is estimated that high frequency traders in the USA nowadays participate in $70 \%$ or more of trades in equities and futures markets. 
situation generates some gaps paving the way to a reciprocal influence between the two fields involved associated in the analogies (F1 $\leftrightarrow \mathrm{F} 2)$.

Giorgio Israel (1996) emphasized such reciprocal influences when he worked on the importance of "mathematical analogies" in science. These analogies are based on the existence of unifying mathematical simple models that are not dedicated to the phenomena studied. Mathematical modeling then uses mathematical analogies by means of which the same mathematical formalism is able to account for heterogeneous phenomena like those in Financial economics and in Physics. These heterogeneous phenomena are "only interconnected by an analogy that is expressed in the form of a common mathematical description" (Israel, 1996, p. 41). The model then is an effective reproduction of reality without ontology, one that may provide an explanation of phenomena. Mathematical analogies illustrate the transfers from Physics to Financial economics, and more generally in Finance, allowing the creation of Econophysics and the extension of knowledge in Physics. In the same vein, these mathematical analogies able also the same kind of transfers but from Financial economics to Physics by suggesting some unknown aspects that physicists gradually integrated into their disciplinary knowledge. It appears that Econophysics is a telling example of the use of "mathematical analogies" in the transfers between Physics and Financial economics ${ }^{15}$ and reciprocally, explaining the key role of Econophysics in the reciprocal influence of these two disciplines.

\section{Conclusion}

By opposition to the traditional influence of Physics on Financial economics, and more generally on Economics, the mathematical analogies and the digitalization of financial data have created a context that allows a mutual influence. The Financial economics, and more generally the Economics, is no longer considered an application field where methods, models and tools from Mathematics and Physics can be used. Econophysicists can use results and open problems from Financial economics in order to explore new challenges in Physics. Even though recent, this methodological trend can be observed in Physics - this paper aims at introducing this moving nature of knowledge between Physics and Financial economics. In this movement, econophysics plays a significant role since it can be looked on as a bridge between the two disciplines. To date, almost all of economists ignore such influence because it has not been studied yet. However, it is very promising. Indeed, a telling illustration of usefulness this influence for financial economists is given by the physicist Dietrich Stauffer who explained that "Once we [the economist Thomas Lux and Stauffer] discussed whether to do a Grassberger-Procaccia analysis of some financial data ... I realized that in this case he, the economist, would have to explain to me, the physicist, how to apply this physics method" (Stauffer, 2004, p. 3).

Understanding this influence could be very profitable for financial economists, and more generally economists. By understanding how their models are currently used for setting new challenges and improving Physics, financial economists will understand

${ }^{15}$ See Schinckus (2018a) for further discussion on this aspect. 
how their theoretical knowledge could provide new perspectives and new horizons for them. It also contributes to shed some light on the current challenges and future developments of Econophysics like those pointed out in Kutner et al. (2019) or Jovanovic and Schinckus (2017).

\section{References}

Akemann, G., Baik, J., \& Di Francesco, P. (Eds.). (2011). The Oxford handbook of randon matrix theory. Oxford: Oxford University Press.

Alastair, B., \& Wallace, D. (1989). Critical phenomena: universal physics at large length scales. In P. Davies (Ed.), The new Physics. Cambridge: Cambridge University Press.

Arous, G. B., \& Guionnet, A. (2008). The spectrum of heavy tailed random matrices. Communications in Mathematical Physics, 278(3), 715-751.

Arthur, B. W. (2004). Foreword to The Minority Game (book of Damien Challet, Matteo Marsili, and Yi-Cheng Zhang).

Arthur, W. B. (1994). Complexity in economic theory: Inductive reasoning and bounded rationality. American Economic Review, 82(2), 406-411.

Ausloos, M., Jovanovic, F., \& Schinckus, C. (2016). On the "usual" misunderstandings between econophysics and finance: Some clarifications on modelling approaches and efficient market hypothesis. International review of financial analysis, 47, 7-14.

Bachelier, L. (1900). Théorie de la spéculation reproduced in. Annales de l'Ecole Normale Supérieure, 3ème série, 17(January), 21-86. Reprint, 1995, J. Gabay, Paris.

Barnett, L., Barrett, A. B., \& Seth, A. K. (2009). Granger causality and transfer entropy are equivalent for Gaussian variables. Physical Review Letters, 103(23), 238701.

Ben-El-Mechaiekh, H., \& Dimand, R. W. (2018). Louis Bachelier's 1938 Monograph on the Calculus of Speculation: Mathematical Finance and Randomness of Asset Prices in Bachelier's later work. Revue d'histoire des mathématiques, 24(1), 41-106.

Bernstein, P. L. (1992). Capital ideas : the improbable origins of modern Wall Street. New York and Toronto: Free Press; Maxwell Macmillan Canada; Maxwell Macmillan International.

Biroli, G., Bouchaud, J.-P., \& Potters, M. (2007). The Student Ensemble of Correlation Matrices: Eigenvalue Spectrum and Kullback--Leibler Entropy. Acta Physica Polonica B, 38(13), 4009-4026.

Blattberg, R., \& Sargent, T. (1971). Regression with Non-Gaussian Stable Disturbances: Some Sampling Results. Econometrica, 39(3), 501-510.

Bouchaud, J.-P., \& Challet, D. (2014). Behavioral finance and financial markets: arbitrage techniques, exuberant behaviors and volatility. opinion et débats, 7 , 20-35.

Bouchaud, J.-P., Mezard, M., \& Potters, M. (2002). Statistical properties of stock order books: Empirical results and models. Quantitative Finance, 2, 251-256.

Bouchaud, J.-P., \& Potters, M. (2011). Financial Applications of Random Matrix Theory: a short review. In G. Akemann, J. Baik, \& P. Di Francesco (Eds.), Handbook on Random Matrix Theory. Oxford: Oxford University Press. 
Boumans, M. (2004). How Economists Model the World into Numbers. London: Routledge.

Brenner, M. (1974). On the stability of distribution of the market component in stock price changes. Journal of Financial and Quantitative Analysis, 9, 945-961.

Buchanan, M. (2012). It's a (stylized) fact! Nature Physics, 8(1), 3.

Bun, J., Bouchaud, J.-P., \& Potters, M. (2017). Cleaning large correlation matrices: tools from random matrix theory. Physics Reports, 666, 1-109.

Burda, Z., Görlich, A., Jarosz, A., \& Jurkiewicz, J. (2004). Signal and noise in correlation matrix. Physica A: Statistical Mechanics and its Applications, 343, 295-310.

Burda, Z., Jurkiewicz, J., Nowak, M. A., Papp, G., \& Zahed, I. (2005). Random Levy Matrices: II. Acta Physica Polonica. Series B, 36(9), 2635-2640.

Casey, M. (2013). Move over economists, markets need physicists. Market Watch Magazine, 10th July.

Chakrabarti, B. K., \& Chakraborti, A. (2010). Fifteen Years of Econophysics Research.

Chakraborti, A., Challet, D., Chatterjee, A., Marsili, M., Zhang, Y.-C., \& Chakrabarti, B. K. (2015). Statistical mechanics of competitive resource allocation using agent-based models. Physics Reports, 552, 1-25.

Challet, D., Marsili, M., \& Zhang, Y.-C. (2013). Minority games: interacting agents in financial markets. New York, NY: Oxford University Press.

Challet, D., \& Zhang, Y.-C. (1997). Emergence of cooperation and organization in an evolutionary game. Physica A, 246(3-4), 407-418.

Cizeau, P., \& Bouchaud, J.-P. (1994). Theory of Lévy matrices. Physical Review E, 50(3), 1810-1822.

Clark, P. K. (1973). A Subordinated Stochastic Process with Finite Variance for Speculative Prices. Econometrica, 41(1), 135-155.

Conlon, T., Ruskin, H. J., \& Crane, M. (2009). Cross-correlations dynamics in financial time series. Physica A, 388(5), 705-714.

Cont, R. (2001). Empirical properties of asset returns: stylized facts and statistical issues. Quantitative Finance, 1, 223-236.

Coolen, T. (2005). The Mathematical Theory of Minority Games: Statistical Mechanics of Interacting Agents. Oxford: Oxford University Press.

De Martino, A., \& Marsili, M. (2006). Statistical mechanics of socio-economic systems with heterogeneous agents. Journal of Physics A: Mathematical and General, 39(43), R465.

Einstein, A. (1905). Uber die von der molekularkinetischen Theorie der Wärme geforderte Bewegung von in ruhenden Flüssigkeiten suspendierten Teilchen. Annalen der Physik, 17, 549-560.

Elmachkour, M., Daha, I., Sabir, E., Kobbane, A., \& Ben-Othman, J. (2014). Green opportunistic access for cognitive radio networks: A minority game approach. Paper presented at the IEEE International Conference on Communications (ICC), Sydney, NSW, Australia.

Elmachkour, M., Sabir, E., Kobbane, A., Ben-Othman, J., \& El Koutbi, M. (2014). The Greening of Spectrum Sensing: A Minority Game-Based Mechanism Design. IEEE Communications Magazine, 52(12), 150-156.

Engle, R. F., \& Russell, J. R. (2004). Analysis of high frequency financial data. In Y. Ait-Sahalia \& L. Hansen (Eds.), Handbook of Financial Econometrics: Tools and Techniques (Handbooks in Finance) (Vol. Volume 1, pp. 383-426). Amsterdam: Elsevier. 
Fama, E. F. (1963a). The distribution of the Daily first differences of stock prices: a test of Mandelbrot's stable Paretian hypothesis. (doctoral dissertation), University of Chicago, Chicago. (unpublished)

Fama, E. F. (1963b). Mandelbrot and the Stable Paretian Hypothesis. Journal of Business, 36(4), 420-429.

Fama, E. F., \& Roll, R. (1968). Some Properties of Symmetric Stable Distributions. Journal of the American Statistical Association, 63(323), 817-836.

Fama, E. F., \& Roll, R. (1971). Parameter Estimates for Symmetric Stable Distributions. Journal of the American Statistical Association, 66(334), 331-338.

Fourcade, M., \& Khurana, R. (2013). From Social Control to Financial Economics: The Linked Ecologies of Economics and Business in Twentieth-Century America. Theory and Society, 42(2), 121-159.

Gabaix, X. (2009). Power Laws in Economics and Finance. Annual Review of Economics, 1, 255-293.

Galstyan, A., Krishnamachari, B., \& Lerman, K. (2004). Resource Allocation and Emergent Coordination in Wireless Sensor Networks. Paper presented at the Workshop on Sensor Networks at the Nineteenth National Conference on Artificial Intelligence, San Jose, California (USA).

Granger, C. W. J. (1969). Investigating causal relations by econometric models and cross-spectral methods. Econometrica, 37(3), 424-438.

Hurwitz, A. (1897). Über die Erzeugung der Invarianten durch Integration. Nachrichten von der Gesellschaft der Wissenschaften zu Göttingen, 71-90.

Ingrao, B., \& Israel, G. (1990). The invisible hand: Economic equilibrium in the history of science London: MIT Press.

Israel, G. (1996). La mathématisation du réel. Essai sur la modélisation mathématique. Paris: Le Seuil.

Jovanovic, F. (2008). The Construction of the Canonical History of Financial economics. History of Political Economy, 40(2), 213-242.

Jovanovic, F. (2012). Bachelier: not the forgotten forerunner he has been depicted as. European Journal for the History of Economic Thought, 19(3), 431-451.

Jovanovic, F., \& Le Gall, P. (2001). Does God practice a random walk? The "financial physics" of a 19th century forerunner, Jules Regnault. European Journal for the History of Economic Thought, 8(3), 323-362.

Jovanovic, F., \& Schinckus, C. (2013a). The History of Econophysics' Emergence: a New Approach in Modern Financial Theory. History of Political Economy, 45(3), 443-474.

Jovanovic, F., \& Schinckus, C. (2013b). Towards a transdisciplinary econophysics. Journal of Economic Methodology, 20(2), 164-183.

Jovanovic, F., \& Schinckus, C. (2017). Econophysics and Financial Economics: An Emerging Dialogue. New York: Oxford University Press.

Kesten, H. (1973). Random difference equations and renewal theory for products of random matrices. Acta Mathematica, 131(1), 207-248.

Kutner, R., Ausloos, M., Grech, D., Di Matteo, T., Schinckus, C., \& Stanley, H. E. (2019). Econophysics and sociophysics: Their milestones \& challenges. Physica A, 516, 240-253

Kutner, R., \& Grech, D. (2008). Report on Foundation and Organization of Econophysics Graduate Courses at Faculty of Physics of University of Warsaw and Department of Physics and Astronomy of the Wrocław University. Acta Physica Polonica A, 114(3), 637-647. 
Laloux, L., Cizeau, P., Bouchaud, J.-P., \& Potters, M. (1999). Noise dressing of financial correlation matrices. Physical Review Letters, 83(7), 1467-1470.

Le Gall, P. (2002). Les Représentations du Monde et les Pensées Analogiques des Economètres. Un Siècle de Modélisation en Perspective. Revue d'Histoire des Sciences Humaines, 6, 39-64.

Lesne, A. (1998). Renormalization Methods. Critical phenomena, Chaos, Fractal Structures. Chichester: John Wiley \& sons.

Lesne, A., \& Laguës, M. (2012). Scale Invariance: From Phase Transitions to Turbulence. Berlin: Springer.

Levy, M., Solomon, S., \& Ram, G. (1996). Dynamical explanation for the emergence of power law in a stock market model. International Journal of Modern Physics C, 7(1), 65-72.

Lux, T. (2009). Applications of Statistical Physics in Finance and Economics. In B. Rosser (Ed.), Handbook of Research on Complexity (pp. 213 - 258). Cheltenham: Edward Elgar.

Maas, H. (2005). William Stanley Jevons and the Making of Modern Economics. New York and Cambridge: Cambridge University Press.

Mähönen, P., \& Petrova, M. (2008). Minority game for cognitive radios: Cooperating without cooperation. Physical Communication, 1, 94-102.

Mandelbrot, B., \& Hudson, R. L. (2004). The (Mis)Behavior of Markets: A Fractal View of Risk, Ruin, and Reward. London: Profile Books.

Mantegna, R. N. (1991). Levy Walks and enhanced diffusion in Milan stock exchange. Physica A, 179(1), 232-242.

Mantegna, R. N. (1999). Hierarchical structure in financial markets. The European Physical Journal B-Condensed Matter and Complex Systems, 11(1), 193-197.

Mantegna, R. N., \& Stanley, H. E. (1999). An introduction to Econophysics: Correlations and Complexity in Finance. New York: Cambridge University Press.

Marinazzo, D., Pellicoro, M., \& Stramaglia, S. (2008). Kernel Method for Nonlinear Granger Causality. Physical Review Letters, 100, 144103.

Markowitz, H. M. (1952). Portfolio Selection. Journal of finance, 7(1), 77-91.

McCauley, J. L. (2006). Response to "Worrying Trends in Econophysics". Physica A, $371(1), 601-609$.

McCauley, J. L. (2009). ARCH and GARCH models vs. martingale volatility of finance market returns. International review of financial analysis, 18(4), 151-153.

McCauley, J. L., Gunaratne, G. H., \& Bassler, K. E. (2007). Martingale option pricing. Physica A, 380, 351-356.

Ménard, C. (1981). La machine et le coeur. Essai sur les analogies dans le raisonnement économique. In A. Lichnerowicz, F. Perroux, \& G. Gadoffre (Eds.), Analogie et connaissance (Vol. vol. 2, chapter 14, pp. 137-161). Paris: Éditions Maloine.

Mézard, M., Parisi, G., \& Virasoro, M. A. (1987). Spin glass theory and beyond: An Introduction to the Replica Method and Its Applications (Vol. Vol. 9). Teaneck, NJ, USA: World Scientific Publishing Company

Mirowski, P. (1989). More heat than light : economics as social physics, physics as nature's economics. Cambridge ; New York: Cambridge University Press.

Mirowski, P. (2012). The Modern Commercialization of Science is a Passel of Ponzi Schemes. Social Epistemology, 2(2), 1-7. 
Morgan, M. S., \& Morrison, M. (Eds.). (1999). Models as mediators: perspectives on natural and social science. Cambridge: Cambridge University Press.

Nagel, E. (1961). The Structure of Science. Problems in the Logic of Explanation. London: Routledge.

Plerou, V., Gopikrishnan, P., Rosenow, B., Nunes Amaral, L. A., \& Stanley, H. E. (1999). Universal and nonuniversal properties of cross correlations in financial time series. Physical Review Letters, 83(7), 1471-1474.

Plerou, V., Gopikrishnan, P., Rosenow, B., Nunes Amaral, L. A., \& Stanley, H. E. (2000). A random matrix theory approach to financial cross-correlations. Physica A, 287, 374-382.

Poitras, G. (2000). The Early History of Financial Economics, 1478-1776. Aldershot (UK): Edward Elgar Publishing.

Poitras, G. (Ed.) (2006). Pioneers of financial economics: Contributions Prior to Irving Fisher (Vol. 1). Cheltenham, UK ; Northampton, MA: Edward Elgar.

Poitras, G., \& Heaney, J. (2015). Classical Ergodicity and Modern Portfolio Theory. Chinese Journal of 1-17. doi:http://dx.doi.org/10.1155/2015/737905

Poitras, G., \& Jovanovic, F. (Eds.). (2007). Pioneers of financial economics: TwentiethCentury Contributions (Vol. 2). Cheltenham, UK ; Northampton, MA: Edward Elgar.

Potters, M., \& Bouchaud, J.-P. (2003). More statistical properties of stock order books and price impact. Physica A, 324, 133-140.

Radicchi, F., Ramasco, J. J., \& Fortunato, S. (2011). Information filtering in complex weighted networks. Physical Review E, 83(4), 046101.

Samuelson, P. A. (1973). Mathematics of Speculative Price. SIAM Review, 15(1), 142.

Schabas, M. (1990). A world ruled by number: William Stanley Jevons and the rise of mathematical economics. Princeton, N.J.: Princeton University Press.

Schinckus, C. (2010a). Econophysics and economics: sister disciplines? American Journal of Physics, 78(1), 325-327.

Schinckus, C. (2010b). Is Econophysics a new discipline? A neo-positivist argument. Physica A, 389, 3814-3821.

Schinckus, C. (2014). Stylized facts and Multiple Realizibility in Econophysics. Result in Physics, 4, 135-136.

Schinckus, C. (2016). 1996-2016: Two decades of econophysics: Between methodological diversification and conceptual coherence. The European Physical Journal, 255(17-18), 3299-3311.

Schinckus, C. (2018a). From DNA to Economics: Analogies in Econobiology. Review of Contemporary Philosophy, 17, 3-17.

Schinckus, C. (2018b). When Physicists Become Undisciplined: An Essay on Econophysics. (Ph.D. dissertation), University of Cambridge, Cambridge.

Serrano, M. Á., Boguná, M., \& Vespignani, A. (2009). Extracting the multiscale backbone of complex weighted networks. Proceedings of the National Academy of Sciences of the United States of America, 106(16), 6483-6488.

Shafique, M., Bauer, L., Ahmed, W., \& Henkel, J. (2011). Minority-Game-based Resource Allocation for Run-Time Reconfigurable Multi-Core Processors. Paper presented at the Design, Automation \& Test in Europe Conference \& Exhibition (DATE), Grenoble, France. 
Sidi, H. B. A., Chahin, W., El-Azouzi, R., De Pellegrini, F., \& Walrand, J. (2013). Incentive Mechanisms based on Minority Game in Heterogeneous DTNs. https://arxiv.org/pdf/1207.6760.pdf.

Sinha, S., Chakrabarti, A. S., \& Mitra, M. (2016). What is economics that physicists are mindful of it? The European Physical Journal Special Topics, 225(17-18), 3087-3089.

Sornette, D. (2013). [How we can predict the next financial crisis].

Sornette, D. (2014). Physics and financial economics (1776-2014): puzzles, Ising and agent-based models. Reports on progress in physics, 77(6), 062001-062028.

Sornette, D., \& Cauwels, P. (2015). Financial Bubbles: Mechanisms and diagnostics. Review of Behavioral Economics, 2(3), 279-305.

Stanley, H. E. (1999). Scaling, universality, and renormalization: Three pillars of modern critical phenomena. Reviews of Modern Physics, 71(2), S358-S366.

Stanley, H. E., Afanasyev, V., Amaral, L. A. N., Buldyrev, S., Goldberger, A., Havlin, S., . . . Viswanathan, K. (1996). Anomalous fluctuations in the dynamics of complex systems: from DNA and physiology to econophysics. Physica $A$, 224(1), 302-321.

Stanley, H. E., Gabaix, X., Gopikrishnan, P., \& Plerou, V. (2006). Statistic Physics and Economic Fluctuations. In L. E. Blume \& S. N. Durlauf (Eds.), The Economy as Evolving Complex System III. Oxford: Oxford University Press.

Stanley, H. E., \& Plerou, V. (2001). Scaling and universality in economics: empirical results and theoretical interpretation. Quantitative Finance, 1(6), 563-567.

Stauffer, D. (2004). Introduction to statistical physics outside physics. Physica A, 336, $1-5$.

Sungwook, K. (2014). Evolutionary Minority Game Model for Congestion Control Scheme. Wireless Personal Communications, 78(2), 1199-1210.

Taqqu, M. S. (2001). Bachelier and his Times: A Conversation with Bernard Bru. Finance and Stochastics, 5(1), 3-32.

Teichmoeller, J. (1971). A note on the distribution of stock price changes. Journal of the American Statistical Association, 66, 282-284.

Tumminello, M., Aste, T., Di Matteo, T., \& Mantegna, R. N. (2005). A tool for filtering information in complex systems. Proceedings of the National Academy of Sciences of the United States of America, 102(30), 10421-10426.

Tumminello, M., Lillo, F., \& Mantegna, R. N. (2007). Kullback-Leibler distance as a measure of the information filtered from multivariate data. Physical Review E, 76(3), 031123.

Tumminello, M., Lillo, F., \& Mantegna, R. N. (2010). Correlation, hierarchies, and networks in financial markets. Journal of economic behavior \& organization, 75(1), 40-58.

Tumminello, M., Micciche, S., Lillo, F., Piilo, J., \& Mantegna, R. N. (2011). Statistically validated networks in bipartite complex systems. PloS one, 6(3), e17994.

Walstad, A. (2010). Comment on " econophysics and economics: Sister disciplines? " by Christophe Schinckus. American Journal of Physics, 78(8), 325-327.

Whitley, R. D. (1986). The Rise of Modern Finance Theory: its characteristics as a scientific field and connection to the changing structure of capital markets. In W. J. Samuels (Ed.), Research in the History of Economic Thought and Methodology (Vol. 4, pp. 147-178). Stanford: JAI Press Inc.

Wigner, E. P. (1957). On the Distribution of the Roots of Certain Symmetric Matrices. Annals of Mathematics, 67(2), 325-327. 
Wishart, J. (1928). The generalized product moment distribution in samples from a normal multivariate population. Biometrika, 20A(1/2), 32-52.

Zhang, C., Wu, W., Huang, H., \& Yu, H. (2012). Fair Energy Resource Allocation by Minority Game: Algorithm for Smart Buildings. Paper presented at the Design, Automation \& Test in Europe Conference \& Exhibition (DATE), Dresden, Germany. 